\title{
Continuous-wave ultrasonic modulation of scattered laser light to image objects in turbid media
}

\author{
Lihong Wang, Steven L. Jacques, and Xuemei Zhao
}

Laser Biology Research Laboratory, Box 17, M. D. Anderson Cancer Center, The University of Texas, 1515 Holcombe Boulevard, Houston, Texas 77030

Received October 19, 1994

\begin{abstract}
Continuous-wave ultrasonic modulation of scattered laser light has been used to image objects in tissue-simulating turbid media for what is to our knowledge the first time. The ultrasound wave focused into the turbid media modulates the laser light passing through the ultrasonic focal zone. The modulated laser light collected by a photomultiplier tube reflects the local mechanical and optical properties in the focal zone. Buried objects are located with millimeter resolution by scanning and detecting alterations of the modulated optical signal. This technique has the potential to provide a noninvasive, nonionizing, inexpensive diagnostic tool for diseases such as breast cancer.
\end{abstract}

Breast cancer is the most common malignant neoplasm and the leading cause of cancer-related deaths of women in the United States. A means for prevention of breast cancer has not been found, and early detection and treatment are the best methods to improve the cure rate. Mammography and ultrasonography are used for detecting breast cancer clinically. However, both methods have known limitations, and additional approaches are highly desirable. Research on nonionizing laser detection of breast cancer, based on the difference between the optical properties of normal and abnormal tissues,,$^{1,2}$ is a new and active field. The current research topics in this area include time-domain short-pulse laser imaging, ${ }^{3-5}$ frequency-domain imaging, ${ }^{6-10}$ and other ballistic or quasi-ballistic imaging. ${ }^{11-14}$ The key problem with these techniques is the trade-off between imaging resolution and signal. ${ }^{15-17}$ Marks et al. have investigated tissue imaging by using the combination of pulsed ultrasound and laser light and have detected the signal of a homogeneous turbid medium without buried objects. ${ }^{18}$

We present a new approach: continuous-wave ultrasound-modulated laser-light imaging (Fig. 1). The major advantage of using continuous-wave ultrasound modulation rather than pulsed ultrasound modulation is the significant increase in signal-tonoise ratio, which allows us to image buried objects in turbid media.

We prepared a liquid tissue phantom (turbid medium) to simulate the optical properties of tissues by dissolving dominantly absorbing Trypan blue dye and dominantly scattering polystyrene spheres $(579 \pm 21 \mathrm{~nm}$ in diameter) in deionized water. The optical properties of the phantom at the 632.8-nm wavelength were absorption coefficient $\mu_{a}=0.01 \mathrm{~cm}^{-1}$, scattering coefficient $\mu_{s}=2.0 \mathrm{~cm}^{-1}$, and anisotropy factor $g=0.853$. The compressibility of the water, $4.6 \times 10^{-5} \mathrm{bar}^{-1}{ }^{19}$ is of the same order of magnitude as that of most tissues ${ }^{20}$ because of the high water content of biological tissues. A solid glass bead (either a cube or a sphere) was suspended at the center of the cuvette from a U-shaped metalwire frame, where the wire holding the bead was a 0.64-mm-diameter metal wire. The glass beads had 1.1-mm diameter holes drilled through their centers. These holes were used as a means of tying the beads to the metal wire. The glass cuvette was seated on a two-dimensional translation stage, which was able to scan the cuvette along both the $x$ and $z$ axes. While the cuvette was scanned, the rest of the system, including the optical and ultrasonic systems, was fixed.

The $\mathrm{He}-\mathrm{Ne}$ laser (Uniphase, 1135P) with $10-\mathrm{mW}$ output power, 632.8-nm wavelength, and $0.68-\mathrm{mm}$ waist diameter delivered a Gaussian beam perpendicular to the left surface of the cuvette. After the aperture, the optical detector, and the ultrasound focal spot were aligned with the laser beam, the phantom solution was poured into the glass cuvette, whose inner dimensions were 15,10 , and $15 \mathrm{~cm}$ along the

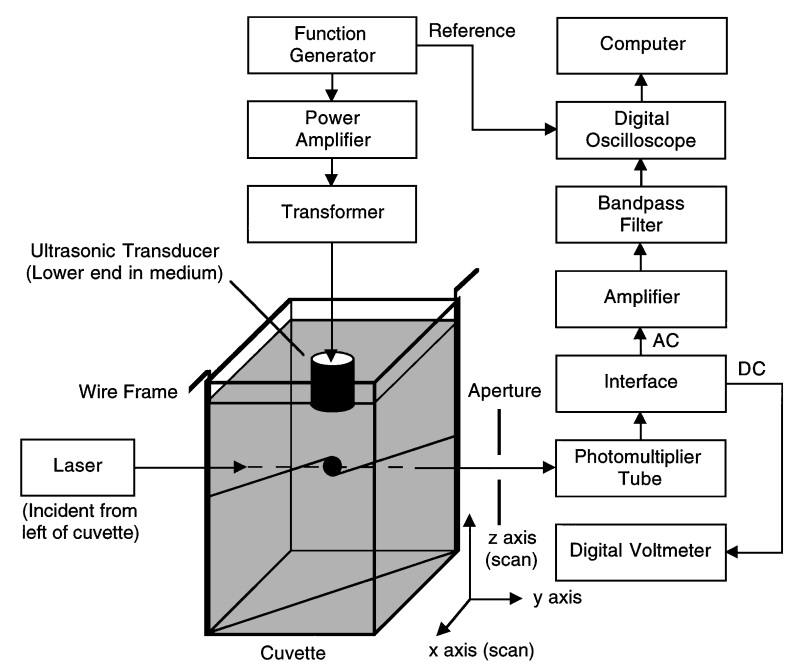

Fig. 1. Setup of imaging of turbid media using ultrasound-modulated scattered laser light. The arrows between blocks represent electrical signals, and the arrows from the laser and from the cuvette represent optical signals. 
$x, y$, and $z$ axes, respectively. The lower end of the ultrasonic transducer was buried in the solution to permit good coupling of the ultrasound wave. The room lights were turned off to reduce the ambient noise collected by the photomultiplier tube (PMT; Hamamatsu, R928). The voltage supply of the PMT (Hamamatsu, HC 123-01) was powered with a $12-\mathrm{V}$ dc power supply, and the voltage of the PMT cathode was set to $-660 \mathrm{~V}$.

The function generator (Stanford Research Systems, DS345) produced a sinusoidal wave at a fixed frequency of $999825 \mathrm{~Hz}$, which was the center frequency of the bandpass filter in the detection system. This signal drove the ultrasonic transducer (Panametrics, V314-SU) after being amplified to 45$\mathrm{V}$ amplitude by the power amplifier and the transformer. The operating bandwidth of the ultrasonic transducer was centered at $1 \mathrm{MHz}$. The diameter of the active element of the ultrasonic transducer was $1.9 \mathrm{~cm}$. The focal length of the transducer in water was $2.54 \mathrm{~cm}$. The diameter of the ultrasound focal spot in water was $0.2 \mathrm{~cm}$. The distance between the exit end of the laser and the cuvette was $40 \mathrm{~cm}$. The distance between the cuvette and the aperture was $5 \mathrm{~cm}$. The distance between the aperture and the PMT was $10 \mathrm{~cm}$. The diameter of the aperture was $1.1 \mathrm{~mm}$.

The design of the above experiment was based on a simple hypothesis. The continuous ultrasound wave was propagated through the turbid solution and focused to a small spot inside the solution. The ultrasound wave near the focal point produced a pressure varying sinusoidally as a function of time. The pressure variation induced a density change in the solution as a result of the compressibility of the solution. The optical absorption and scattering coefficients were proportional to the number density of absorbers and scatterers, respectively. The index of refraction was also related to the density. Therefore the density modulation in turn modulated the optical properties of the solution at the ultrasound frequency and hence modulated the light passing through the focal zone.

The modulated light carried the information of the optical properties and compressibility near the focal spot. After light passed through the aperture and reached the PMT, the optical signal was converted into an electrical signal. The electrical signal was separated into dc and ac components by an interface circuit. The dc voltage was read by a digital voltmeter, and the ac voltage was amplified and then effectively filtered by a narrow-bandpass filter. The filtered signal was collected and averaged over 256 sweeps by the digital oscilloscope (Tektronix, 2440 ), which was triggered by a reference signal from the function generator. Both frequency filtering and signal averaging enhanced the signal-to-noise ratio. The averaged time-domain signal was then transferred to a Macintosh computer for data storage and processing. The peak-to-peak voltage of the signal represented the light signal modulated by the ultrasound wave.

Once the time-domain signal in the oscilloscope was stored in the computer, the cuvette was moved to the next $(x, z)$ position, and the above steps were repeated. When the signals of all the scanned positions were obtained, a one- or two-dimensional image of the turbid medium containing a buried object was constructed.

We observed a two-dimensional image of a black glass cube with a contrast of approximately $98 \%$, using the ultrasound-modulated light signal (Fig. 2). The translation stage was scanned at $1 \mathrm{~mm}$ per step in both the $x$ and the $z$ directions. The cube was placed in the center of the cuvette, replacing the position of the buried sphere in Fig. 1. The facets of the cube were parallel to those of the cuvette, and the dimensions of the cube along the $x, y$ and $z$ axes were $8.8,8.8$, and $7.9 \mathrm{~mm}$, respectively. When the object was away from the ultrasound focus, the signal was strong. When the object was moved toward the ultrasound focus, the signal dropped quickly to the noise level.

To demonstrate that this technique is able to differentiate objects of partial absorption from those of complete absorption, we buried two glass spheres (beads) individually inside the cuvette containing the turbid medium that was used for the previous experiment. The two spheres included a completely absorbing black sphere and a partially absorbing blue sphere, and the diameter of each sphere was $8 \mathrm{~mm}$. Each sphere was positioned at the center of the cuvette along the $x$ and $y$ axes. The heights of the cuvette and the glass sphere were adjusted such that the center of the sphere was aligned with the laser beam and the focal spot of the ultrasound field.

The normalized one-dimensional images of the two spheres obtained by use of both the dc signals and the ac signals are shown in Fig. 3, where we set the center of the $x$ axis to the center of the object to align the two images. The dc signals of both spheres showed a small dip when the spheres were at the ultrasound focal spot, where the signals at the dip were approximately $65 \%$ of the background signals. The difference between the two dc dips was small.

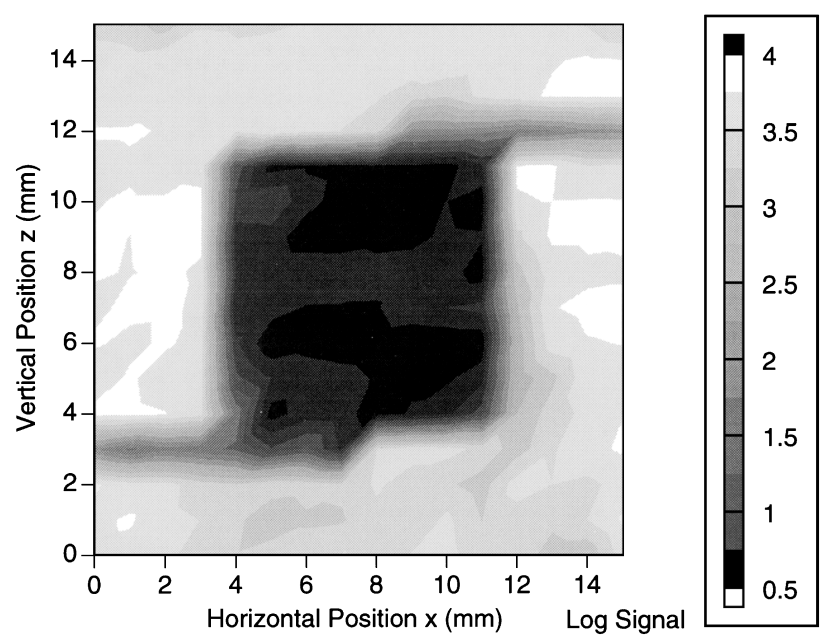

Fig. 2. Two-dimensional image of a buried glass cube plotted on a logarithmic scale of the peak-to-peak voltage signals of the ultrasound-modulated light in millivolts. The holding wire on the top right and bottom left of the cube was observed at the same time. 


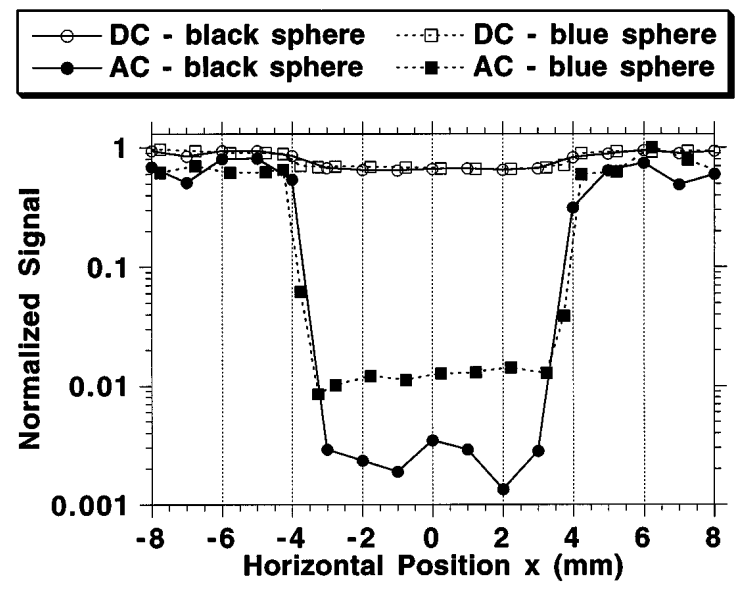

Fig. 3. One-dimensional images of a black and a blue glass sphere obtained with the dc unmodulated laser-light signals and the ac ultrasound-modulated laser-light signals.

The ac signals of both spheres showed a steep dip when the spheres were at the ultrasound focal spot, where the dip of the black sphere was deeper than that of the blue sphere. The imaging resolution for both spheres was approximately $1 \mathrm{~mm}$, which was close to one half of the 2-mm focal-spot size of the ultrasound. The imaging contrasts of the black and blue spheres were $99 \%$ and $98 \%$, respectively.

The light entering the black object, even though it was modulated by the ultrasound wave, was trapped and could not escape to reach the detector. Therefore the ac signal of the black sphere should have been determined by the system background noise. The light passing through the blue sphere was modulated by the ultrasound wave, and some of the modulated light was collected by the detector. Therefore the blue sphere showed a larger ac signal at the dip than did the black sphere.

Because both glass spheres were made of the same material with different additive dyes for the colors, they should have had the same mechanical properties. This experiment demonstrates that the ultrasound-modulated laser-light imaging technique is able to detect optical properties for objects possessing the same mechanical properties, whereas pure ultrasound imaging cannot differentiate the partially absorbing glass sphere from the completely absorbing black sphere. This experiment also demonstrated the dramatic contrast enhancement of imaging that uses the ac ultrasound-modulated light over the dc unmodulated light.

The acoustic impedance of glass is 8.7 times that of water. ${ }^{21}$ Tumors and normal tissues may not always differ so much mechanically. A rubber cube, whose acoustic impedance was measured to be approximately 1.3 times that of water, was imaged similarly without degradation of the contrast or resolution compared with the images of the glass objects.

These studies have demonstrated the feasibility of the ultrasound-modulated laser-light imaging of buried objects in tubid media. If clinical applications of this technique succeed, tumors can be imaged and differentiated from surrounding normal tissues based on optical and mechanical properties by use of nonionizing radiation.

We thank L. Eppich for proofreading the manuscript and D. Liu for experimental assistance. This research was supported in part by the University of Texas M. D. Anderson Cancer Center, the Whitaker Foundation, the U.S. Air Force Office of Scientific Research, the U.S. Department of Energy, and the National Institutes of Health. Correspondence may be addressed to L. Wang (phone 713-792-3664).

\section{References}

1. W. F Cheong, S. A. Prahl, and A. J. Welch, IEEE J. Quantum Electron. 26, 2166 (1990).

2. V. G. Peters, D. R. Wyman, M. S. Patterson, and G. L. Frank, Phys. Med. Biol. 35, 1317 (1990).

3. L. Feng, K. M. Yoo, and R. R. Alfano, Appl. Opt. 32, 554 (1993).

4. J. C. Hebden and D. T. Delpy, Opt. Lett. 19,311 (1994).

5. S. Andersson-Engels, R. Berg, S. Svanberg, and O. Jarlman, Opt. Lett. 15, 1179 (1990).

6. B. Chance, K. Kang, L. He, J. Weng, and E. Sevick, Proc. Natl. Acad. Sci. (USA) 90, 3423 (1993).

7. B. J. Tromberg, L. O. Svaasand, T. T. Tsay, and R. C. Haskell, Appl. Opt. 32, 607 (1993).

8. J. B. Fishkin and E. Gratton, J. Opt. Soc. Am. A 10, 127 (1992).

9. M. A. O'Leary, D. A. Boas, B. Chance, and A. G. Yodh, Phys. Rev. Lett. 69, 2658 (1992).

10. A. Knuttel, J. M. Schmitt, R. Barnes, and J. R. Knutson, Rev. Sci. Instrum. 64, 638 (1993).

11. E. Leith, C. Chen, H. Chen, Y. Chen, D. Dilworth, J. Lopez, J. Rudd, P. C. Sun, J. Valdmanis, and G. Vossler, J. Opt. Soc. Am. A 9, 1148 (1992).

12. M. R. Hee, J. A. Izatt, E. A. Swanson, and J. G. Fujimoto, Opt. Lett. 18, 1107 (1993).

13. M. D. Duncan, R. Mahon, L. L. Tankersley, and J. Reintjes, Opt. Lett. 16, 1868 (1991).

14. L.-H. Wang, D. V. Stephens, A. H. Hielscher, F. K. Tittel, and S. L. Jacques, in Advances in Optical Imaging and Photon Migration, R. R. Alfano, ed., Vol. 21 of OSA Proceedings Series (Optical Society of America, Washington, D.C., 1994), p. 288.

15. J. A. Moon, R. Mahon, M. D. Duncan, and J. Reintjes, Opt. Lett. 18, 1591 (1993).

16. L.-H. Wang and S. L. Jacques, in Advances in Optical Imaging and Photon Migration, R. R. Alfano, ed., Vol. 21 of OSA Proceedings Series (Optical Society of America, Washington, D.C., 1994), p. 181.

17. S. L. Jacques, L.-H. Wang, and A. H. Hielscher, in Optical Thermal Response of Laser Irradiated Tissue, A. J. Welch and M. J. C. van Gemert, eds. (Plenum, New York, 1995)

18. F. A. Marks, H. W. Tomlinson, and G. W. Brooksby, Proc. Soc. Photo-Opt. Instrum. Eng. 1888, 500 (1993).

19. D. R. Lide, ed., CRC Handbook of Chemistry and Physics, 73rd ed. (CRC, Boca Raton, Fla., 1992), p. 6114.

20. F. A. Duck, Physical Properties of Tissue; A Comprehensive Reference Book (Academic, New York, 1990), Chap. 5, p. 160.

21. L. E. Kinsler and A. R. Frey, Fundamentals of Acoustics, 3rd ed. (Wiley, New York, 1982), Chap. A10, p. 461. 